\title{
Optical Tomography for Biomedical Applications
}

\author{
Lihong V. Warng, Ph.D., Optical Imaging Laboratory, Texas A\&M University
}

$\mathrm{M}$ edical imaging has revolutionized the practice of medicine in the past century. Physicians are empowered to "see" through the human body for abnormalities noninvasively and to make diagnostic decisions rapidly, which has impacted the therapeutic outcomes of the detected diseases.

Medical imaging dates back to 1895 when X-rays were discovered serendipitously by German physicist W.C. Roentgen, who received the first Nobel Prize in physics in 1901 (http://www.almaz.com/nobel/) for this important work. Human anatomy can be easily imaged by simple X-ray projections, such as chest $\mathrm{X}$ rays, which are still being used in the clinic. Contemporary medical imaging began with the invention of computerized tomography $(\mathrm{CT})$ in the 1970 s. G.N. Hounsfield in England produced the first computer-recor/structed images experimentally based on the theoretical founcation laid by A.M. Cormack in the United States. Both of them were awarded the Nobel Prize in medicine in 1979. The essence of CT is that if an object is viewed from a number of different angles, then a cross-sectional image of it can be computed, i.e., "reconstructed."

The advent of CT has inspired other new tomographic and even 3-D imaging techniques. The application of reconstruction to conventional nuclear medicine imaging led to positron emission tomography (PET) and single photon emission computed tomography (SPECT). A similar application to the technique of nuclear magnetic resonance led to magnetic resonance imaging (MRI).

Different imaging modalities are used to detect different aspects of biological tissues through a variety of contrast mechanisms. X-ray imaging primarily detects electron. In MRI, proton density and its associated relaxation properties are detected. Using ultrasonography, acoustic impedance is detected. In nuclear imaging, nuclear racliation emitted from the body is detected after introducing a radiopharmaceutical inside the body to tag a specific biochemical function.

The advantages and disadvantages of different imaging modalities may be illustrated using breast cancer detection. Breast cancer is the most common malignant neoplasm and the leading cause of cancer deaths in women in the United States. A means for prevention of breast cancer has not been found, and early detection and treatmert is the best solution to improve cure rate. At present, X-ray mammography and ultrasonography are clinically used for breast cancer detection. Mammography is currently the only reliable means of detecting nonpalpable breast cancers. As a supplementary tool, ultrasound is used to evaluate the internal matrix of circumscuibed masses found using mammography or of palpable masses that are obscured by radiographically dense parenchyma using nhammography. However, X-ray mammography is ionizing radiation, and imaging of radiographically dense breasts is difficult. Ultrasonography cannot detect many of the nonpalpable cancers that are not visible on mammograms of

good quality. Most of the studies claiming that ultrasonography is useful in this regard compare it to poor-quality mammography or fail to compare it to mammography.

Several other techniques are under investigation for breast cancer imaging. Magnetic resonance imaging offers great promise for imaging of the radiographically dense breast. Breast MRI is superior to mammography in differentiating solid from cystic lesions and is equivalent to mammography in providing information regarding different parenchymal patterns. Injection of intravenous contrast material with MRI increases cancer detectability in spite of the fact that breast cancer and glandular tissues have similar magnetic resonance tissue characteristics. However, breast MRI is expensive, has inferior spatial resolution to mammography, and cannot image microcalcifications. Breast CT has also been investigated for the differentiation of benign from malignant solid masses. Breast CT involves the use of intravenous injection of iodinated contrast material and has limited spatial resolution and high cost; hence, it is not suited for routine breast cancer screening.

Nonionizing optical tomography is a new and active research field although projection-light imaging was investigated as early as 1929. The optical properties of normal and diseased tissues are usually different despite the large variation of values in optical properties of the normal tissues alone. Therefore, it is possible to detect some breast cancers based on measurements of optical properties.

The optical difference is not surprising because cancerous tissues manifest significant architectural changes at the cellular and subcellular levels, and the cellular components that cause elastic scattering have dimensions typically on the order of visible to near-IR wavelengths. Some tumors are associated with vascularization, where blood causes increased light absorption. The use of optical contrast agents may also be exploited to enhance the optical contrast between normal and abnormal tissues. Because the optical information is determined by the molecular conformations of biological tissues, optical imaging is expected to provide sensitive signatures for early cancer detection and monitoring.

Because tissues are optically turbid media that are highly scattering, light is quickly diffused inside tissues as a result of frequent scattering. The strong scattering has made optical detection of biological tissues challenging. A typical scattering coefficient for visible light in biological tissues is $100 \mathrm{~cm}-1$ in comparison with $0.2 \mathrm{~cm}-1$ for X-rays used in medical diagnostics. Light transmitted through tissues is classified into three categories: ballistic light, quasi-ballistic light, and diffuse light. Ballistic light experiences no scattering by tissue and thus travels straight through the tissue. Ballistic light carries direct imaging information as X-ray radiation does. Quasi-ballistic light experiences minimal scattering and carries some imaging information. 
Multiply-scattered diffuse light carries little direct imaging information and overshadows ballistic or quasi-ballistic light.

One of the techniques used for optical tomography is called "early-photon imaging." If diffuse light is rejected, and ballistic or quasi-ballistic light is collected, buried objects can be detected much like X-ray projection. This technique uses a short-pulse laser (ps pulse width) to illuminate the tissue. Only the initial portion of transmitted light is allowed to pass to a light detector, and the latearriving light is gated off by a fast optical gate. Because the ballistic or quasi-ballistic photons travel along the shortest path length, they arrive at the detector sooner than diffuse photons. If only ballistic light is detected, the technique is called ballistic imaging. It has been shown that ballistic imaging is feasible only for tissue of thickness less than $1.4 \mathrm{~mm}$ or 42 mean free paths. Most ballistic imaging techniques reported in the literature have achieved approximately $30 \mathrm{mfp}$. Therefore, this approach is suitable for thin tissue samples but suffers loss of signal and resolution for thick tissues as a result of the strong scattering of light by the tissue.

For tissue of clinically useful thickness $(5$ to $10 \mathrm{~cm}$ ), scattered light must be used to image breast cancers. We have shown that for a 5 -cm-thick breast tissue with an assumed absorption coefficient of $0.1 \mathrm{~cm}^{-1}$ and a reduced scattering coefficient of $10 \mathrm{~cm}^{-1}$, the detector must collect transmitted light that has experienced at least 1,100 scattering events in the tissue to yield enough signal. Therefore, ballistic light or even quasi-ballistic light does not exist for practical purposes. However, if a $10-\mathrm{mW}$ visible or nearinfrared laser is incident on one side of the $5-\mathrm{cm}$ thick breast tissue, we have estimated using diffusion theory that the diffuse transmittance is on the order of $10 \mathrm{nW} / \mathrm{cm}^{2}$ or 1010 photons $/\left(\mathrm{s} / \mathrm{cm}^{2}\right)$, which is detectable using a photomultiplier tube capable of single-photon counting. Similarly, the diffuse transmittance through a $10-\mathrm{cm}$ thick breast tissue would be on the order of $1 \mathrm{pW} / \mathrm{cm}^{2}$ or 106 photons $\left(\mathrm{s} / \mathrm{cm}^{2}\right)$. The significant transmission of light is due to the low absorption coefficient despite the high scattering coefficient.

Imaging resolution of pure laser imaging degrades linearly with increased tissue thickness. The temporal profiles of the scattered light may be detected using a streak camera. The early portion of the profiles was integrated to construct the images of buried objects in a turbid medium. This time-domain technique requires expensive short-pulse lasers and fast light detectors.

Another technique for laser light imaging is the frequencydomain technique, also called photon-density wave imaging. This technique uses amplitude-modulated laser light (at approximately $100 \mathrm{MHz}$ ) to illuminate the tissue and detects the diffuse light. This technique has similar imaging resolution as the timedomain technique but usually requires complex imaging reconstruction algorithms.

Ultrasound-modulated optical tomography is a relatively new technique. An ultrasonic wave focused into the turbid media modulates the laser light passing through the ultrasonic field. The modulated laser light collected by a photomultiplier tube reflects primarily the local mechanical and optical properties in the zone of ultrasonic modulation. A raster scanning over a heterogeneous turbid medium yields an image of the medium based on the ultrasound-modulated optical signal. This technique depends on diffuse light, where the ultrasound adds imaging information to the diffuse light.

Optical techniques may also measure the optical spectra of biological tissues noninvasively. Besides the absorption spectra that are usually measured for other materials, the scattering spectra may also be measured. The optical spectra may be used to quantify important physiological parameters such as the saturation of hemoglobin oxygenation.

In summary, optical techniques have the following advantages: (1) the use of nonionizing radiation, (2) the capability of measuring functional (physiological) parameters, (3) the potential high sensitivity to pathologic state of biological tissues, and (4) low cost. However, biomedical optics is a challenging research field requiring the participation of many diverse, talented scientists and engineers.

The following references are provided for further readings on these optical tomographic approaches.

- B. Chance and R.R. Alfano, eds., Optical Tomography and Spectroscopy of Tissue: Theory, Instrumentation, Model, and Human Studies II, Proc. Soc. of Photo-Opt. Instrum. Eng. 2979 (SPIE Press, Bellingham, WA, 1997).

- W. Cai, B.B. Das, F. Liu, M. Zevallos, M. Lax, and R.R. Alfano, "Time-resolved optical diffusion tomographic image reconstruction in highly scattering turbid media," Proceedings of the National Academy of Sciences of the United States of America 93, 13561-13564 (1996).

- J.B. Fishkin and E. Gratton, "Propagation of photon density waves in strongly scattering media containing an absorbing semi-infinite plate bounded by a straight edge,"J. Opt. Soc. of Am. A 10, 127-134 (1992).

- X.D. Li, T. Durduran, A.G. Yodh, B. Chance, and D.N. Pattanayak, "Diffraction tomography for biochemical imaging with diffuse-photon density waves," Optics Letters 22, 573575 (1997).

- L.-H. Wang, S.L. Jacques, and X.-M. Zhao, "Continuouswave ultrasonic modulation of scattered laser light to image objects in turbid media," Optics Letters 20, 629-631 (1995).

Lihong $V$. Wang, Ph.D. can be reached at the Optical Imaging Laboratory, Biomedical Engineering Program, Texas A\&M University, College Station, Texas 77843-3120, USA. Tel: 409847-9040; E-mail: LWang@tamu.edu;URL: http://biomed.tamu.edu/ $/ \mathrm{Iw}$. 